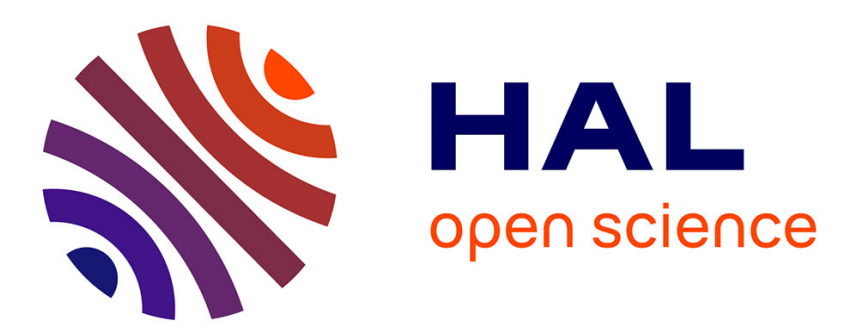

\title{
Typologie et caractérisation des scories de réduction de la métallurgie du fer
}

Cécile Le Carlier de Veslud, Alain Ploquin

\section{To cite this version:}

Cécile Le Carlier de Veslud, Alain Ploquin. Typologie et caractérisation des scories de réduction de la métallurgie du fer. Archeosciences, revue d'Archéométrie, 1999, 23, pp.59-69. hal-02356195

\section{HAL Id: hal-02356195 \\ https://hal-univ-rennes1.archives-ouvertes.fr/hal-02356195}

Submitted on 26 Apr 2020

HAL is a multi-disciplinary open access archive for the deposit and dissemination of scientific research documents, whether they are published or not. The documents may come from teaching and research institutions in France or abroad, or from public or private research centers.
L'archive ouverte pluridisciplinaire HAL, est destinée au dépôt et à la diffusion de documents scientifiques de niveau recherche, publiés ou non, émanant des établissements d'enseignement et de recherche français ou étrangers, des laboratoires publics ou privés. 


\title{
TYPOLOGIE ET CARACTÉRISATION DES SCORIES DE REDUCTION DE LA METALLURGIE DU FER
}

\author{
Cécile MAHÉ-LECARLIER* et Alain PLOQUIN*
}

\begin{abstract}
Résumé : Depuis une quinzaine d'années, l'étude des déchets de la métallurgie ancienne a pris une réelle ampleur grâce à la réalisation d'une large banque de données d'analyses chimiques. L'étude pétrographique présentée ici, associée à ces données a permis de proposer une nouvelle typologie des scories de réduction des minerais de fer. Trois grands types ont été définis : les scories de type I regroupant les laitiers et les quasi-laitiers, provenant aussi bien de hauts que de bas fourneaux, les scories de type II et III proviennent de bas fourneaux, les dernières étant considérées comme les déchets classiques des bas fourneaux, alors que le type II présente des caractères intermédiaires entre les types I et III. Ce type d'étude permet en outre de différencier l'utilisation d'un minerai calcique de l'ajout de castine à un minerai non calcique en bas fourneau.
\end{abstract}

\begin{abstract}
Over the last fifteen years, the study of paleometallurgical slags has significantly advanced thanks to the development of a large data bank of chemical analyses. The petrographical study of these slags leads to proposing a new typology of slags formed during the iron ore's reduction. Three types have been proposed : in the type I, named "laitier" and "quasi-laitier", slags come from blast furnaces or bloomery furnaces. Types II and III are made in bloomery furnaces. Type III represents classical slags of bloomery furnaces while type II is intermediate between types I and III. This work allows, moreover, to differentiate the use of a calcic ore from to the addition of lime to a non-calcic ore in bloomery furnaces.
\end{abstract}

Mots-clés : Paléométallurgie, scorie, pétrographie, chimie, typologie, haut fourneau, bas fourneau.

Key-worlds : Archaeometallurgy, slag, petrography, chemistry, typology, blast furnace, bloomery furnace.

\section{INTRODUCTION}

L'étude des déchets de la métallurgie ancienne a pris une réelle ampleur depuis une quinzaine d'années, notamment avec la réalisation d'une banque de données d'analyses chimiques (PAL-SID) concernant des déchets, essentiellement de la sidérurgie : des scories, des parois de four et des minerais. Depuis quelques années, l'accent est également mis en direction de la caractérisation pétrographique des scories.

Dans cette étude, nous proposons de nous intéresser aux scories de réduction de minerais de fer. L'étude macroscopique, microscopique et chimique de ces déchets a permis de proposer une nouvelle typologie des scories de réduction des minerais de fer.

Depuis quelques années, Ploquin (1994) et Ploquin et al., (1996) ont avancé l'idée qu'il fallait dissocier le concept de procédés techniques de celui de processus physico-chimiques. Les procédés techniques concernent les différents types de fours de réduction (bas et hauts fourneaux, etc...), d'affinage et de forgeage qui se sont succédés au cours des âges. Les processus physico-chimiques concernent les réactions (dont fusion et cristallisation) se déroulant dans ces fours. Ces réactions dépendent de la température, des pressions partielles en gaz (dont notamment l'oxygène, le monoxyde carbone ou l'acide carbonique) et de la composition chimique du bain fondu. Tous ces facteurs peuvent varier fortement et rapidement dans un même appareil et dans une même opération. Comme nous le constaterons lors de la description pétrographique et chimique, il existe des scories présentant des phases cristallisées semblables, provenant aussi bien de bas et de hauts fourneaux. Ainsi, des processus physico-chimiques identiques peuvent avoir lieu dans des fours de types différents (haut fourneau récent, haut fourneau archaïque ou bas fourneau), conduisant à la production de déchets très similaires.

Après avoir donné quelques rappels sur l'évolution de la sidérurgie, sur les filières techniques et sur les techniques d'analyses et d'observation, nous présenterons glo- 
balement la typologie avant de donner les caractères macroscopiques, minéralogiques et chimiques de chaque type de scories. Dans une dernière partie, l'utilisation des minerais calciques ou l'ajout de castine sont mis en évidence par les analyses chimiques.

\section{1 - ÉVOLUTION DE LA SIDÉRURGIE}

Les tout premiers objets en fer connus proviennent du Moyen-Orient (Iran, Mésopotamie, Anatolie et Egypte) (Waldbaum, 1980). La métallurgie y aurait débuté il y a environ 4000 ans par l'utilisation du fer météorique et du fer tellurique natifs. Aux IX-VIIIème siècles avant notre ère, le fer devient d'un usage fréquent au Moyen-Orient. A partir de là, plusieurs points de vue existent quant à la propagation de cette technologie. Selon le point de vue «diffusioniste», qui est actuellement parfois remis en cause, l'influence, par l'intermédiaire des grecs et des phéniciens se fera en direction des Balkans, du sud de l'Italie, en Espagne et au centre de L'Europe par le Caucase. Vers 500 avant J.-C., au Nord des Alpes, le fer devient un métal commun. Les premiers objets en fer atteignent alors seulement les îles britanniques et la Scandinavie.

Au cours des derniers siècles avant notre ère, le fer est produit sur une grande échelle dans de nombreuses régions d'Europe mais toujours par la méthode directe de réduction, dans des bas fourneaux (fig. 1). A la période gauloise la réduction du fer était bien contrôlée, comme le montre par exemple, la taille et la complexité des fours trouvés en fouille aux Clérimois, près de Sens (Dunikowsky et Cabboï, 1995) et dont le mode d'emploi n'est pas encore entièrement élucidé (reconstitutions expérimentales à l'Archéodrome de Beaune-Tailly, juillet 1995 et août 1996). C'est avec l'avènement du procédé indirect que la production va connaître un développement quantitatif sans précédent.

C'est à la suite du développement des applications de l'énergie hydraulique aux machines qu'apparaît en Europe le haut fourneau (fig. 2). Le passage du bas-fourneau au haut-fourneau a été situé par les archéologues vers le XIIIXIVème siècle (Tylecote, 1987; Benoit et al., 1991; Ploquin, 1993) voire au XIIème siècle en Suède (Magnusson, 1996) et en Allemagne (Knau, 1994). En effet, à partir de cette époque beaucoup de scories retrouvées et datées correspondent à ce type de procédé, mais on peut supposer une coexistence du bas fourneau et du haut fourneau avec des appareils intermédiaires (Stücköfen, Massöfen, Flussöfen, etc...). L'emploi de cette nouvelle technologie est généralisée à la fin du XVIème siècle (Belhoste, 1986), malgré la survie de forges catalanes ou Evenstad (Scandinavie) jusqu'au XIXème siècle. Le haut fourneau introduit un changement radical. Outre le fait qu'il augmente considérablement le rendement du minerai, il permet de traiter des volumes considérables de matières premières. La chaîne opéra-

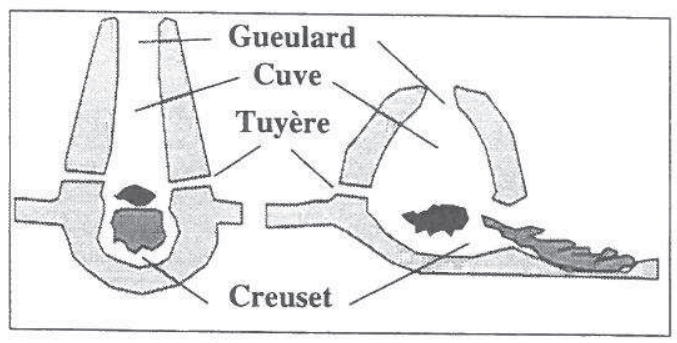

Fig. 1 : Coupes schématiques de deux des principaux types de bas fourneaux (d'après Serneels, 1993). toire est largement modifiée. Depuis le XIIIème siècle, les techniques se sont améliorées : on a décuplé le rendement par une augmentation dans la taille des fours, par une plus grande puissance des souffleries et le réchauffement de l'air insufflé, et surtout par l'introduction du coke dont la tenue mécanique a permis la construction de four de plus grande taille, mais aussi par l'amélioration des techniques d'affinage de la fonte et de l'acier, etc... Au XXème siècle, le développement du convertisseur permet d'obtenir de l'acier en grande quantité.

\section{2-RAPPELSURLES FILİEES TECHNIOUES DE RÉDUCTIONET PRÉSENTATION DELA TYPOLOGIE}

Lors de la phase de réduction, il faut distinguer deux filières. La filière directe où le fer peu carburé est obtenu sous forme d'éponge ou loupe. L'extraction de la loupe hors du four conduit, dans la plupart des cas, à une destruction au moins partielle de celui-ci. La filière indirecte où le produit sortant du four est une fonte liquide qui subira ultérieurement une phase de décarburation, dite d'affinage, afin d'obtenir soit de l'acier, soit du fer doux. En général, à la filière directe est associé le bas fourneau, et à la filière indirecte, le haut fourneau.

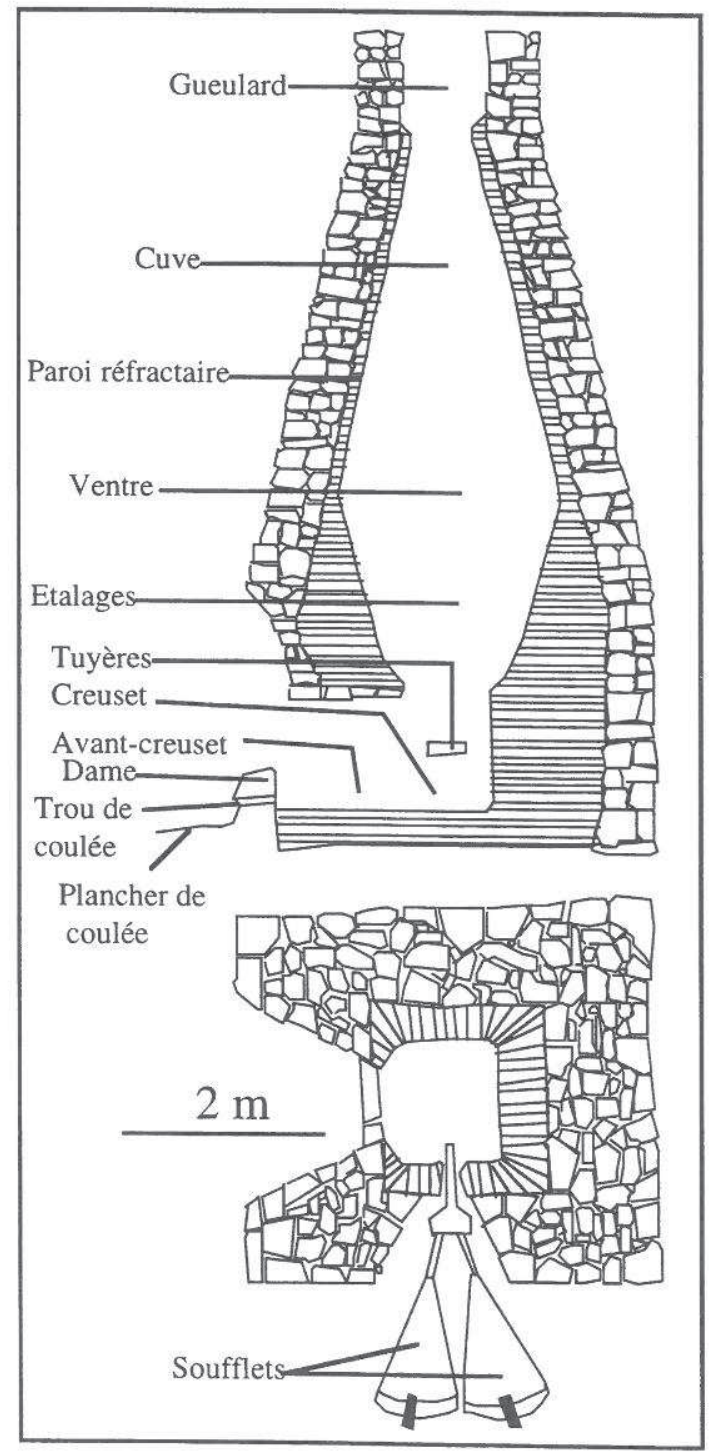

Fig. 2.: Schéma d'un haut fourneau au charbon de bois (XVIIXVIII $^{\text {ème }}$ siècle) (d'après G.S.A.F., 1991). 


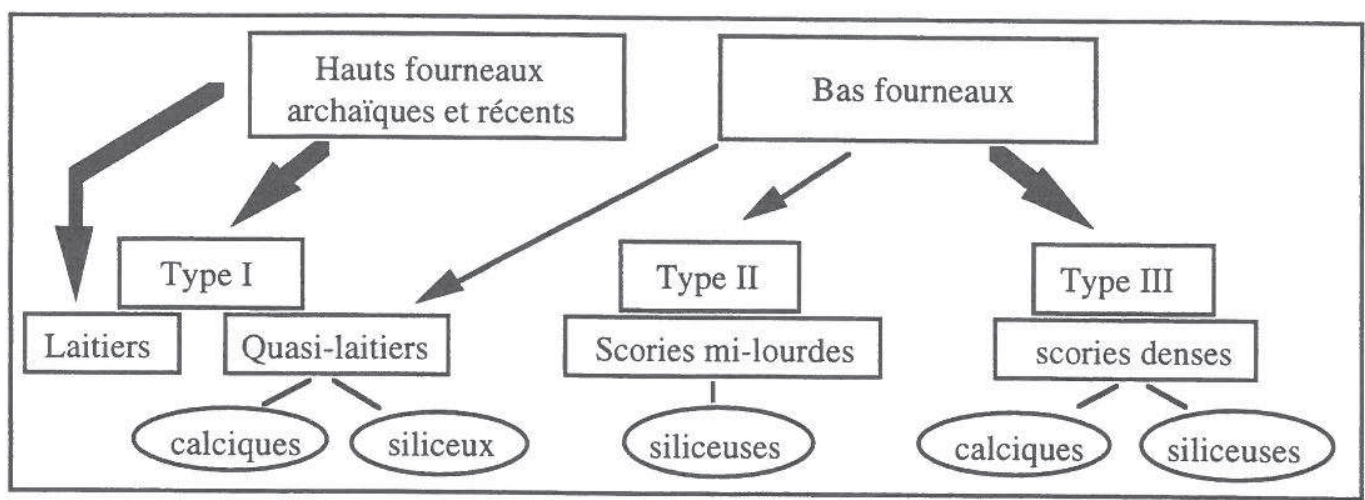

Fig. 3 : Schéma de la typologie et lien avec le type de four.

Soulignons que nous avons exclu de cette étude les laitiers hypercalciques des hauts fourneaux modernes (au coke, fort ajout de castine, haute température).

Trois types de scories ont été définies sans prendre en compte l'époque de fabrication (fig. 3). Le type I regroupe les laitiers et les quasi-laitiers. Le type III rassemble les scories denses, et enfin le type II que l'on peut considérer intermédiaire aux deux premiers. Il est très banal d'associer systématiquement les scories denses de types III et II aux bas fourneaux et les laitiers de type I aux hauts fourneaux. Ceci est vrai dans la très grande majorité des cas. Pourtant, des exceptions existent, tels que les laitiers de Oulches (Mahé-Le Carlier et al., 1998).

L'influence du type de four est nette, car les laitiers et quasi-laitiers de type I dans les bas fourneaux sont associés à des fours de grandes dimensions alors que les scories denses de type III sont formées dans des fours de toutes dimensions. Les scories de type II, qui présentent des caractères intermédiaires entre les scories de type I et les scories de type III, sont également formées dans des fours d'assez grandes dimensions.

Les scories de type I se différencient en plusieurs soustypes que l'on peut relier au type de four. Il y a les laitiers récents ou laitiers de hauts fourneaux pré-modernes, les laitiers anciens ou laitiers de hauts fourneaux archaïques, les laitiers de bas fourneaux et les quasi-laitiers de bas fourneaux. Les scories de type II trouvés étudiés jusqu'à présent sont unitypes. Les scories de type III se différencient en deux sous-types en fonction de leur composition chimique, les scories calciques et les scories siliceuses.

\section{3 - SITES CHOISIS POUR CETTE PRÉSENTATION}

Pour l'étude pétrographique, un certain nombre de sites ont été retenus. Les laitiers pré-modernes proviennent du site de Savignac-Lédrier (Dordogne, environ 1900-Dubois et al., 1992). Les laitiers de hauts fourneaux archaïques proviennent du même site (entre 1575 et 1640 sur ${ }^{14} \mathrm{C}$ ) et de celui de Rogéville (Meurthe et Moselle, sans doute XVIème siècle-Leroy, 1993). Les laitiers de bas fourneaux proviennent du site de Oulches (Indre, entre le troisième quart du IIİ̀me siècle et le troisième quart du IVème siècleDieudonné-Glad, 1991) et les quasi-laitiers viennent des sites de Frouard (Meurthe et Moselle, fin de l'époque galloromaine-Vème siècle après J.-C.-Leroy, 1993 ; Leroy et al., 1994), Bellaires III (Jura Vaudois Suisse, deux fourneaux du site de Bellaires III sont datés du Haut Moyen Age-VIVII siècle-Serneels, 1993) et Les Carolines (Jura Vaudois Suisse, Serneels, 1993).
Les scories de type II proviennent du site de Oulches. Les scories calciques du type III viennent du site de Frouard, celles siliceuses, du site de Oulches et d'une expérimentation à Villejuif.

Pour l'étude chimique, des scories d'autres sites ont été prises en compte. Ce sont les sites de Moulaine (actuel, Lorraine) pour les hauts foumeaux actuels, Paimpont (Illeet-Vilaine, XVIIIème) pour les hauts fourneaux récents, Pas du Houx (llle-et-Vilaine, XVIème), Lapphytan (Suède, XIIXVIème), Articole (Isère, XVIIIème), Bordogna (Lombardie, XV-XVIIème), Sexey-aux-Forges et Tremblecourt (Meurthe et Moselle, XVIème) pour les hauts fourneaux archailques, Manoncourt et Ludres (Meurthe et Moselle, VIIVIIIème) pour les quasi-laitiers calciques de bas fourneaux, Les Martys (Montagne Noire, Ier avant J.-C.-IIIème après J.-C.) et Les Clérimois (Yonne, les grands fours : Ier avant J.-C.-IIème après J.-C.) pour les scories de type II et les scories de type III.

\section{4-MÉTHODES D'ANALYSES}

Les échantillons sont dans un premier temps examinés macroscopiquement. Un premier classement peut dès lors être effectué. Ensuite, ils sont sciés, une partie servant à la confection des lames minces, une seconde étant broyée pour l'analyse chimique, et une dernière étant conservée en tant que témoin.

Minéralogie. Des diffractogrammes de $\mathrm{RX}$ réalisés sur poudres permettent d'évaluer la nature des phases cristallines présentes en faisant référence au fichier ASTM (American Society for Testing Materials). Pour l'observation microscopique par transmission et par réflexion, des lames d'épaisseur $30 \mu \mathrm{m}$ ont été réalisées. Celles-ci ont également servi pour les micro-analyses au moyen de la microsonde électronique (Cameca SX-50, Faculté des Sciences de Nancy) équipée de quatre spectromètres et d'un analyseur dispersif en longueur d'onde (WDS). Les analyses sont effectuées sous une tension d'accélération de $15 \mathrm{kV}$ et un courant de $10 \mathrm{nA}$. Les éléments analysés sont $\mathrm{Si}, \mathrm{Al}, \mathrm{Fe}, \mathrm{Mn}, \mathrm{Mg}, \mathrm{Ca}, \mathrm{Na}, \mathrm{K}, \mathrm{Ti}, \mathrm{P}$. Les standards sont effectués sur des échantillons géologiques avant chaque séance d'analyse.

Chimie. Dans un premier temps, les échantillons préparés sont broyés de manière à obtenir des grains inférieurs à $70 \mu \mathrm{m}$. La méthode analytique est la suivante : $100 \mathrm{mg}$ de poudre d'échantillon sont fondus dans un creuset en platine avec $900 \mathrm{mg}$ de métaborate de lithium et $600 \mathrm{mg}$ d'acide borique. Le mélange obtenu est ensuite dissout dans de l'acide nitrique. Les solutions sont alors analysées par ICP-MS et ICP-AES. Les éléments majeurs ( $\mathrm{Si}, \mathrm{Al}, \mathrm{Fe}, \mathrm{Mn}$, 
$\mathrm{Mg}, \mathrm{Ca}, \mathrm{Na}, \mathrm{K}, \mathrm{Ti}$, et $\mathrm{P}$ ) sont analysés par spectrométrie d'émission atomique couplée à une induction plasma (ICPAES), tandis que les éléments en traces sont obtenus par spectrométrie de masse couplée à une induction plasma (ICP-MS). Les appareils utilisés sont un Jobin-Yvon $70 \mathrm{~T}$ II pour l'ICP-AES et un Perkin Elmer élan 5000 pour l'ICPMS (CRPG, Nancy).

\section{5 - CARACTĖRES MACROSCOPIQUES}

Les scories de type I : Les laitiers les plus récents étudiés ici sont âgés de 100 ans environ et peuvent être cristallisés, d'aspect pierreux, de couleur grise et plus ou moins vacuolaires (photo 1). Ils peuvent être aussi en partie vitreux et leur teinte est alors très variable. Nous retiendrons trois exemples : des laitiers dont la zone cristallisée est blanche grisâtre et le verre violet noir (photo 2), des laitiers à zone cristallisée brune noire et à verre vert bouteille, des laitiers à zone cristallisée brune et à verre vert pâle. Ces trois exemples de laitiers ne présentent qu'une faible porosité.

Les laitiers plus anciens, provenant de hauts fourneaux archaïques (XVIème siècle environ), sont plus souvent vitreux de couleur vert bouteille. Quand ils sont cristallisés, leur couleur est blanc grisâtre. La grande caractéristique de ces laitiers archaïques est leur grande hétérogénéité (photo 3), particulièrement au niveau de l'abondance et de la taille des vacuoles, de la présence et de la taille de billes de métal, et des impuretés qui sont souvent de couleur blanchâtre.

\begin{tabular}{|c|l|l|}
\hline T I & $\begin{array}{l}\text { laitiers } \\
\text { quasi- } \\
\text { laitiers }\end{array}$ & $\begin{array}{l}\text { *billes de fer, fer automorphe } \\
\text { *wüstite absente } \\
\text { *pyroxène Al, wollastonite, feldspath } \\
\text { *reliques de minerais } \\
\text { *spinelle, olivine ou mélilite dans } \\
\text { quasi-laitier }\end{array}$ \\
\hline T II & $\begin{array}{l}\text { scories } \\
\text { mi- } \\
\text { lourdes }\end{array}$ & $\begin{array}{l}\text { *billes de fer } \\
\text { *wüstite très rare (ou localisée) } \\
\text { *souvent spinelle } \\
\text { *olivine }\end{array}$ \\
\hline T III & $\begin{array}{l}\text { scories } \\
\text { denses }\end{array}$ & $\begin{array}{l}\text { *fer amiboïde, parfois automorphe } \\
\text { *wüstite omniprésente } \\
\text { *olivine ou mélilite }\end{array}$ \\
\hline
\end{tabular}

Tab. 1 : Caractères minéralogiques principaux des trois types reconnus.
Les laitiers trouvés sur les sites à bas fourneaux ressemblent beaucoup aux laitiers récents avec éventuellement une partie cristallisée grise (photo 4) contenant de nombreuses vacuoles et une partie vitreuse allant du vert bouteille au bleu turquoise.

Du point de vue macroscopique, les quasi-laitiers siliceux se différencient des laitiers par leur abondance en grandes vacuoles, faisant de ces scories des matériaux très peu denses et très vacuolaires. Leur couleur est brune noire, d'aspect un peu terreux (photo 5). Les scories qualifiées de «scories noires vitreuses» par Serneels (1993) correspondent pour la plupart à notre appellation de quasilaitiers. Les «scories vitreuses» sont formées par de petites coulées en cordons, sont extrêmement vitreuses avec une très faible porosité. Les quasi-laitiers calciques sont très vitreux et présentent une porosité extrêmement faible.

Les scories de type III sont les scories denses classiques, décrites de nombreuses fois, en particulier Jarrier (1993), Leroy (1993), Leroy et Ploquin (1994), Jarrier et al., (1997) (photo 6). Ce sont des scories de couleur noire. Leur morphologie est directement influencée par le mode de fonctionnement du four (accumulation interne, écoulement hors du four, etc...). Les scories coulées hors du four sont formées par une superposition de petits cordons de coulées de 1 à $3 \mathrm{~cm}$ de large.

Les scories de type II ne se différencient pas macroscopiquement des scories de type III, sinon qu'elles sont moins denses (photo 6). La différence est à peine perceptible. En fait, sauf grande expérience, la reconnaissance de ce type n'est vraiment possible que sous le microscope.

\section{6- CARACTÈRES MINÉRALOGIQUES}

A partir d'observations en lames minces, sous microscope optique en lumière transmise et en lumière réfléchie, on note des similitudes dans l'assemblage qualitatif des phases minérales (tab. 1) pour un type de scories.

Le fer élémentaire participe à quatre types de phases : le fer métallique et la wüstite (oxyde de fer FeO) (tab. 2), les spinelles et les silicates. Le fer métallique se présente toujours sous forme de billes dans les types I et II et sous forme amiboïde (photo 7) ou en section quelconque arrondie dans le type III. La forme automorphe du système cubique (photo 7) peut être parfois présente dans le type I et III. Le fer métallique peut apparaître selon deux processus bien distincts : fusion de l'ensemble de la charge en un bain fondu puis séparation du fer métallique soit par

\begin{tabular}{|c|c|c|c|c|c|c|c|c|c|}
\hline & wüstite & $\begin{array}{c}\text { spinelle } \\
\text { hercynite } \\
-2-\end{array}$ & $\begin{array}{c}\text { spinelle } \\
\text { magnétite } \\
-3-\end{array}$ & $\begin{array}{c}\text { clino-px } \\
\text { baguette- } \\
4-\end{array}$ & $\begin{array}{c}\text { clino-px- } \\
\text { gd plume } \\
-5-\end{array}$ & $\begin{array}{c}\text { clino-px- } \\
\text { ptt-plume } \\
-6-\end{array}$ & $\begin{array}{c}\text { clino-px } \\
\text { baguette- } \\
7-\end{array}$ & $\begin{array}{c}\text { clino-px- } \\
\text { gd plume } \\
\text { - }\end{array}$ & $\begin{array}{c}\text { clino-px- } \\
\text { ptt-plume } \\
-9-\end{array}$ \\
\hline $\mathrm{SiO} 2$ & 1,47 & 1,45 & 0,31 & 53,34 & 54,35 & 49,44 & 53,11 & 51,59 & 53,33 \\
$\mathrm{~A} 2 \mathrm{O} 3$ & 0,96 & 45,45 & 20,84 & 4,27 & 7,46 & 10,41 & 1,61 & 2,05 & 5,43 \\
$\mathrm{FeOt}$ & 90,08 & 46,02 & 7,53 & 0,12 & 0,25 & 0,06 & 7,54 & 8,07 & 8,85 \\
$\mathrm{MnO}$ & 0,70 & 4,25 & 1,91 & 0,65 & 0,86 & 0,71 & 0,86 & 0,76 & 0,71 \\
$\mathrm{MgO}$ & 1,27 & 0,61 & 0,42 & 17,46 & 14,59 & 15,97 & 12,54 & 11,90 & 7,88 \\
$\mathrm{CaO}$ & 1,60 & 1,35 & 0,64 & 22,98 & 20,79 & 23,00 & 24,04 & 23,88 & 20,61 \\
$\mathrm{Na} 2 \mathrm{O}$ & 0,00 & 0,00 & 0,00 & 0,02 & 0,00 & 0,00 & 0,00 & 0,00 & 0,07 \\
$\mathrm{~K} 2 \mathrm{O}$ & 0,02 & 0,03 & 0,02 & 0,05 & 0,66 & 0,18 & 0,02 & 0,02 & 1,68 \\
$\mathrm{TiO} 2$ & 0,59 & 0,36 & 1,26 & 0,80 & 0,36 & 0,63 & 0,39 & 0,48 & 0,50 \\
$\mathrm{P} 205$ & 0,09 & 0,23 & 0,00 & - & - & - & - & - & - \\
\hline total & 96,78 & 99,75 & 95,93 & 99,69 & 99,32 & 100,40 & 100,11 & 98,75 & 99,06 \\
\hline
\end{tabular}

Tab. 2 : Exemples de compositions chimiques de différentes phases cristallines, -1-2-3- scorie de type III de Frouard; -4-5-6- laitier récent de type I de Savignac Lédrier ; -7-8-9- laitier de bas fourneau de type I de Oulches ; «-») : non dosé. 

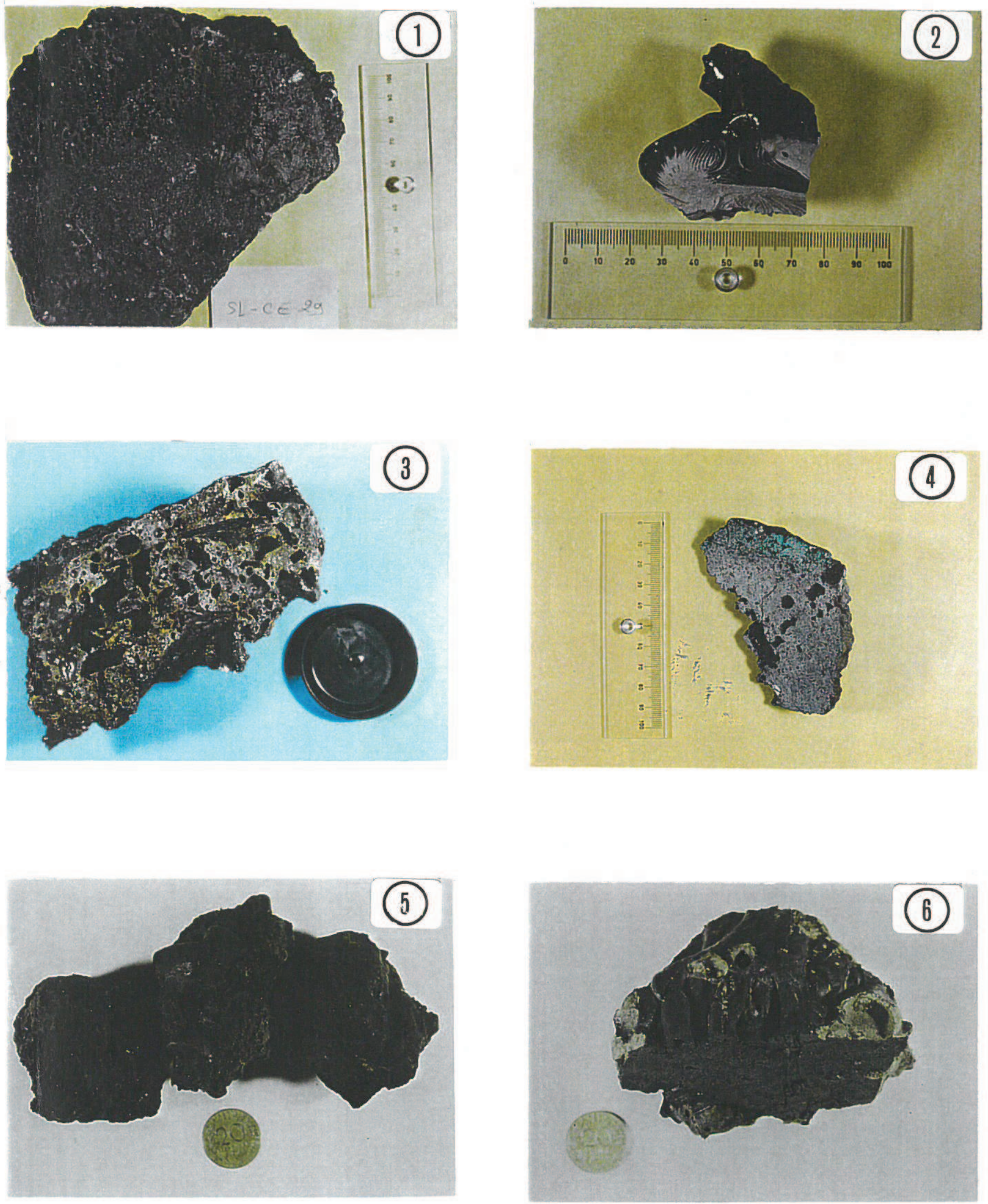

Photos : 1- Laitier de haut fourneau récent gris cristallisé, Savignac-Lédrier (Dordogne-XXe) - 2- Laitier de haut fourneau récent vitreux noir et cristallisé blanc, Savignac-Lédrier (Dordogne-XXe) - 3- Laitier hétérogène de haut fourneau archaïque, Savignac-Lédrier (Dordogne-XVIe) - 4- Laitier de bas fourneau gris cristallisé et blanc vitreux, Oulches (Berry-IV-Ve) - 5- Quasi-laitier siliceux de bas fourneau (Les Carolines, Suisse, VI-VIIe) - 6- Aspect typique des scories écoulées de type II et type III- Ici, scorie de type II de bas fourneau de Oulches (IV-Ve). 

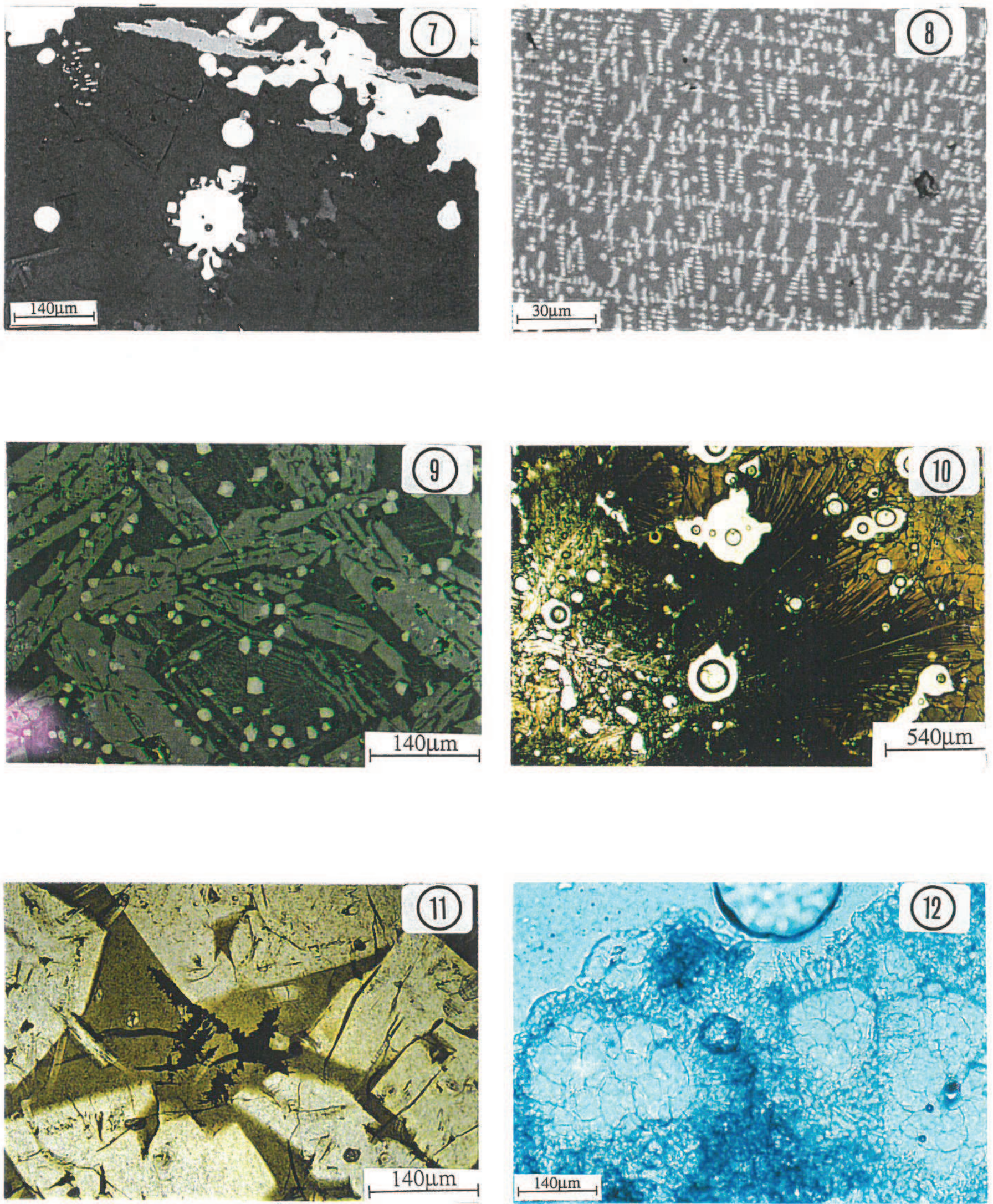

Photos : 7- Scorie de type III calcique de Frouard, fer métal sous forme amiboïde, de billes ou automorphe à section cubique - 8- Scorie de type III siliceux de Oulches, wüstite dendritique en réseau tridimentionnel dans une matrice vitreuse - 9- Scorie de type II de Oulches, petits cristaux automorphes de spinelle (blanc) et lattes de fayalite, dans une matrice vitreuse (noir) - 10- Laitier de haut fourneau récent de savignac-Lédrier, cristaux transparents en baguette de clinopyroxène passant en continuité à des clinopyroxènes en lattes formant des plumes - 11- Scorie de fond de four de type III de Frouard, cristaux automorphes de mélilites (grand cristaux blancs) et cristaux d'apatite (blancs en lattes ou en petites sections hexagonales) dans une matrice vitreuse - 12- Laitier de haut fourneau archaïque de Savignac-lédrier, globules de cristobalite et cristaux de cristobalite dendritiques poussant dessus. 
démixtion, soit par cristallisation, ou réduction des oxydes de fer à l'état solide. La présence de fer sous forme de billes, de cristaux automorphes cubiques ou de dendrites confirment la première hypothèse. Lorsque le fer se présente sous forme amiboïde ou sous forme de globules plus ou moins arrondis, la mise en évidence du processus est plus délicate. La wüstite (photo 8) n'est jamais présente dans le type I (ou très exceptionnellement), rarement présente mais localisée dans le type II et toujours présente dans le type III. Ce sont des dendrites qui se forment également à partir du bain fondu.

Le spinelle (photo 9) n'est présent dans le type I que dans les quasi-laitiers, et n'a jamais été observé dans les laitiers. Il est fréquent dans les types II et III. Le spinelle fait toujours partie de la série continue hercynite-magnétite $\mathrm{FeAl}_{2} \mathrm{O}_{4}-\mathrm{Fe}_{3} \mathrm{O}_{4}$ (tab. 2). Cette phase, lorsqu'elle est présente, se forme après l'apparition du fer métallique mais avant la cristallisation de la wüstite. De la gahnite a été signalée.

Les phases silicatées appartiennent à cinq groupes minéralogiques : les clinopyroxènes, les mélilites, les wollastonites, les olivines et les feldspaths. Ils sont tous présents dans les scories de type I. Mais dans une coulée, généralement un seul type de phase est présent à la fois. Les olivines et les mélilites sont présentes dans les scories de type III, les olivines se développant dans les scories relativement siliceuses, les mélilites dans les scories plus calciques. Nous n'avons rencontré actuellement que des olivines dans les scories de type II.

\begin{tabular}{|c|c|c|c|c|c|}
\hline & $\begin{array}{c}\text { quasi- } \\
\text { laitier } \\
-10-\end{array}$ & $\begin{array}{c}\text { fond } \\
\text { de } \\
\text { four } \\
-11-\end{array}$ & $\begin{array}{c}\text { fond } \\
\text { de } \\
\text { four } \\
-12-\end{array}$ & $\begin{array}{c}\text { fond } \\
\text { de } \\
\text { four } \\
-13-\end{array}$ & $\begin{array}{c}\text { scorie } \\
\text { dense } \\
-14-\end{array}$ \\
\hline $\mathrm{SiO} 2$ & 25,89 & 29,29 & 32,17 & 35,69 & 33,57 \\
$\mathrm{~A} 2 \mathrm{O} 3$ & 30,78 & 20,11 & 15,46 & 6,98 & 11,11 \\
$\mathrm{FeOt}$ & 0,60 & 7,25 & 8,42 & 14,70 & 10,95 \\
$\mathrm{MnO}$ & 0,10 & 0,00 & 0,24 & 0,34 & 0,25 \\
$\mathrm{MgO}$ & 1,98 & 2,15 & 2,82 & 2,35 & 2,37 \\
$\mathrm{CaO}$ & 41,00 & 39,60 & 38,31 & 37,17 & 38,06 \\
$\mathrm{Na} 2 \mathrm{O}$ & 0,10 & 0,28 & 0,41 & 0,34 & 0,39 \\
$\mathrm{~K} 2 \mathrm{O}$ & 0,05 & 0,15 & 0,38 & 0,65 & 0,54 \\
$\mathrm{TiO} 2$ & 0,00 & 0,00 & 0,01 & 0,05 & 0,01 \\
$\mathrm{P} 2 \mathrm{O} 5$ & - & 0,68 & 0,56 & 0,61 & 2,56 \\
total & 100,6 & 99,51 & 98,78 & 98,88 & 99,81 \\
\hline
\end{tabular}

Tab. 3 : Exemple de composition chimique de mélilites de scories de type I (quasi-laitier) et de type III du site de Frouard.
Les clinopyroxènes représentent la phase cristallisée dominante dans les laitiers (tab. 2). Ils se présentent sous plusieurs formes selon la vitesse de cristallisation. Ainsi, au centre de la scorie où le refroidissement est le plus lent, les cristaux ont pratiquement leur forme propre, c'est-àdire qu'ils sont quasiment automorphes, en baguette (photo 10). En se rapprochant de la bordure de la coulée, les cristaux prennent la forme de lattes squelettiques de plus en plus petites et qui se rassemblent en gerbes. Ces dernières ressemblent très fortement à des plumes lors de l'observation en lumière transmise (photo 10). Lorsque les clinopyroxènes sont automorphes, ils présentent une composition de type augite $(\mathrm{Ca}, \mathrm{Mg}) \mathrm{Si}_{2} \mathrm{O}_{6}$. Par contre, lorsqu'ils deviennent de plus en plus dendritiques, ils incorporent dans leur réseau cristallin de plus en plus d'aluminium (tab. 2) donnant une composition d'augite alumineuse. Tous ces cristaux sont calciques et peuvent être soit magnésiens, soit ferriferes, soit les deux.

Lorsque la scorie est beaucoup plus riche en $\mathrm{Ca}$, le clinopyroxène est remplacé par de la mélilite quand les teneurs en aluminium sont très importantes ou de la wollastonite pour des teneurs plus faibles. Dans un cas très manganésiferre, Articole, c'est de la bustamite.

Les mélilites sont classées à partir de quatre pôles : ferroåkermanite ( $\left.\mathrm{Ca}_{2} \mathrm{FeSi}_{2} \mathrm{O}_{7}\right)$, ferro-géhlénite ( $\left.\mathrm{Ca}_{2} \mathrm{FeAlSiO} 7\right)$, géhlénite $\left(\mathrm{Ca}_{2} \mathrm{Al}_{2} \mathrm{SiO}_{7}\right)$, åkermanite $\left(\mathrm{Ca}_{2} \mathrm{MgSi}_{2} \mathrm{O}_{7}\right)$. Des analyses de mélilite pour trois types de scories sont données dans le tableau 3. Dans les scories à dominante vitreuse, les cristaux sont automorphes. Dans les fonds de four (scorie de type III) qui ont refroidi plus lentement, les cristaux sont aussi en grande majorité automorphe (photo 11). Par contre dans les scories denses qui ont coulé à l'extérieur du four, les cristaux se présentent en lattes squelettiques, fort ressemblant aux lattes des clinopyroxènes.

Les wollastonites se forment lorsque le bain fondu n'est pas assez riche en $\mathrm{Al}$ pour permettre la cristallisation des mélilites (tab. 4). Ces cristaux se présentent soit sous la forme de plumes (comme pour les clinopyroxènes) lorsque la scorie est essentiellement cristallisée, soit sous forme de baguette automorphe isolée lorsque la scorie est essentiellement vitreuse.

Les compositions des olivines sont très proches du pôle ferrifère, la fayalite (tab. 4), parfois plus calciques, parfois plus manganésifère. Dans les scories de types II et III, ces cristaux forment de grandes lattes squelettiques (photo 9) se rassemblant souvent en gerbes dont la base se localise soit au niveau des surfaces de coulées, soit au bord de vacuoles. Dans les quasi-laitiers siliceux de type I, les cris-

\begin{tabular}{|c|c|c|c|c|c|c|}
\hline & $\begin{array}{c}\text { wollastonite } \\
-15-\end{array}$ & $\begin{array}{c}\text { olivine } \\
-16-\end{array}$ & $\begin{array}{c}\text { olivine } \\
-17-\end{array}$ & $\begin{array}{c}\text { feldspath } \\
-18-\end{array}$ & $\begin{array}{c}\text { feldspath } \\
-19-\end{array}$ & $\begin{array}{c}\text { apatite } \\
-20-\end{array}$ \\
\hline $\mathrm{SiO} 2$ & 50,16 & 29,88 & 29,89 & 49,08 & 58,41 & 6,05 \\
$\mathrm{Al} 2 \mathrm{O} 3$ & 0,03 & 0,10 & 0,14 & 28,76 & 6,04 & 0,22 \\
$\mathrm{FeOt}$ & 0,09 & 65,50 & 60,18 & 0,29 & 5,08 & 1,02 \\
$\mathrm{MnO}$ & 0,00 & 1,14 & 7,40 & 0,08 & 3,04 & 0,08 \\
$\mathrm{MgO}$ & 0,08 & 1,41 & 1,24 & 1,73 & 0,14 & 0,04 \\
$\mathrm{CaO}$ & 47,94 & 0,34 & 0,36 & 17,31 & 24,65 & 55,58 \\
$\mathrm{Na} 2 \mathrm{O}$ & 0,00 & 0,00 & 0,00 & 0,55 & 0,48 & 0,01 \\
$\mathrm{~K} 2 \mathrm{O}$ & 0,08 & 0,00 & 0,00 & 0,45 & 0,51 & 0,04 \\
$\mathrm{TiO} 2$ & 0,00 & 0,14 & 0,08 & 0,05 & 0,13 & 0,00 \\
$\mathrm{P} 2 \mathrm{O} 5$ & 0,78 & - & 0,00 & 0,16 & 0,56 & 34,72 \\
\hline total & 99,16 & 98,51 & 99,29 & 98,46 & 99,05 & 97,76 \\
\hline
\end{tabular}

Tab. 4 : Exemples de compositions chimiques de différentes phases cristallines : -15 -laitier de type I de haut fourneau archaĩque de Rogéville; -16-quasi-laitier de type I de bas fourneau de Bellaires III ; -17-scorie de type II de Oulches ; -18-laitier de type I de haut fourneau archaïque de Savignac Lédrier; -19-laitier de type I de haut fourneau archaïque de Lapphyttan; -20-scorie de type III de Frouard. 
taux présentent la même zonation et le même aspect que les clinopyroxènes, avec au centre de coulée des baguettes évoluant petit à petit vers des grandes plumes puis des petites plumes en se rapprochant de la surface supérieure de coulée.

La dernière phase silicatée que nous avons rarement rencontré est un feldspath (tab. 4) qui se présente sous forme de baguette. La leucite (feldspathoïde potassique) a été signalée dans des scories denses (Jarrier, 1993).

Une phase particulière a été rencontrée dans les scories issues de la réduction de la Minette de Lorraine : l'apatite (photo 11). C'est un phosphate riche en $\mathrm{Ca}$ (tab. 4) qui se forme grâce à la grande richesse en phosphore de ce type de minerai calcique. Il se rencontre aussi bien dans les quasi-laitiers de type I que dans les scories de type III. Cette observation nous amène à nuancer l'une des conclusions de Ph. Dillmann (in Meyer-Roudet dir., 1999) à savoir, l'apatite pourrait caractériser, dans le métal, les inclusions scoriacées formées lors de l'affinage de la fonte.

Dans les trois types de scories, les phases minérales sont toujours noyées dans une matrice vitreuse. Dans les scories de type I, celle-ci est très pauvre en fer pour des laitiers récents, plus riche en fer pour des laitiers de hauts fourneaux archaïques et de bas fourneaux (fig. 4). L'abondance du fer dans la phase vitreuse est encore plus élevée dans les scories de type II. Par contre, dans les scories de type III, cette teneur en fer chute nettement, surtout si on la compare à celle du type II. En effet, dans ce dernier type de scories, il y a formation en premier lieu de dendrites de wüstite qui consomment une très grande quantité de fer du bain fondu, ce qui n'est pas le cas dans les scories de type II.

Les scories de type I provenant de hauts fourneaux archaïques ou de bas fourneaux sont caractérisées par la présence de reliques de minerais non entièrement fondus. Ce sont généralement des grains de quartz $\left(\mathrm{SiO}_{2}\right)$ partiellement ou totalement transformés en cristobalite tuilée et globularisée sur lesquelles se sont développés des cristaux dendritiques de cristobalite (photo 12). Entre ces globules et dendrites, des myriades de minuscules billes de fer métallique semblent avoir été piégées.

Comme cette description microscopique a pu le montrer, des phases minérales peuvent être caractéristiques d'un type de scorie, telle la wüstite pour le type III. Mais, pour

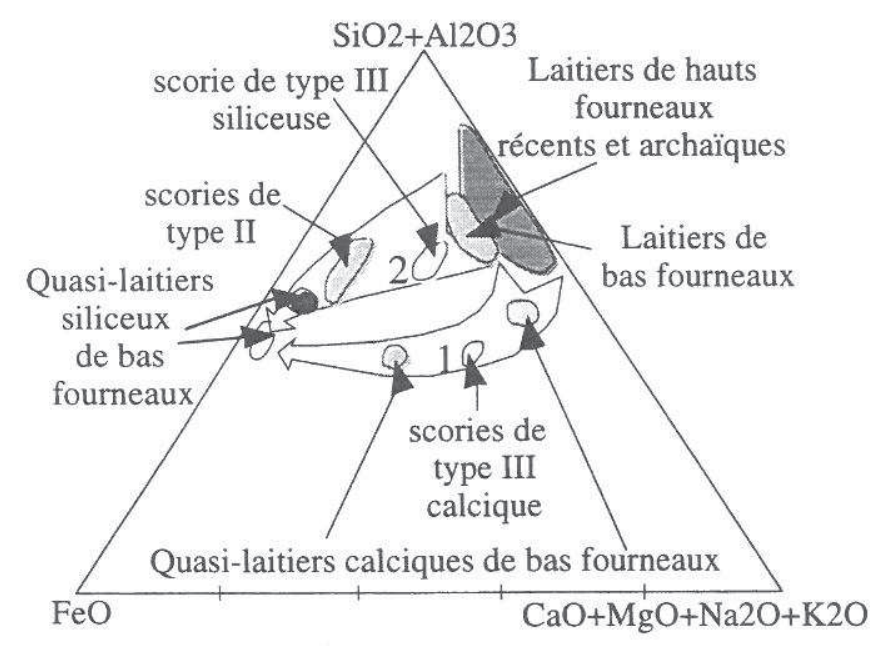

Fig. 4 : Evolution de la composition chimique de la phase vitreuse résiduelle. -1- scories calciques; -2 - scories siliceuses. la majeure partie des phases, ce n'est pas un caractère déterminant, comme par exemple pour l'olivine qui peut s'observer dans les trois types de scories.

Les caractères déterminants résultent du recoupement des observations macroscopiques et microscopiques, et notamment dans ce dernier cas, des associations minérales:

Les scories de type I sont formées par de grosses coulées (plusieurs centimètres de diamètre) dans lesquelles une phase minérale silicatée forme une zonation de par la forme des individus (automorphe au centre puis de plus en plus dendritiques jusqu'à la bordure qui est vitreuse). Le fer métallique a été fondu avec la masse du liquide avant de se séparer de celle-ci soit par démixtion, soit par cristallisation. Il peut exister des restes de minerai si la température du four n'était pas suffisante et si le temps de présence dans le four n'était pas assez important.

Les scories écoulées de type III sont formées par une succession de petits cordons ( 1 à $2 \mathrm{~cm}$ de diamètre) dans lesquels plusieurs phases minérales se succèdent (oxydes puis silicates). Une zonation de taille et de forme des cristaux n'est quasiment pas visible du fait du faible diamètre des cordons. Le processus de formation du fer métallique n'est pas bien connu, sauf pour les scories issues de la réduction de la Minette de Lorraine où la réduction à l'état solide a été fossilisée grâce à la présence de fantômes d'oolithes.

Les scories écoulées de type II sont formées, comme beaucoup de scories de type III, par une succession de petits cordons. La présence du fer métal uniquement sous forme de billes et l'absence de wüstite rapproche ce type de scories du type I. On peut donc parler de scories intermédiaires entre les deux autres types.

\section{7 - COMPOSITION CHIMIQUE GLOBALE}

Une sélection de compositions chimiques est donnée dans le tableau 5. Dans le type I, elle met en évidence une grande variation des teneurs en $\mathrm{SiO}_{2}, \mathrm{Al}_{2} \mathrm{O}_{3}, \mathrm{FeO}, \mathrm{MgO}$ et $\mathrm{CaO}$. Le nombre de cas actuellement reconnus ne permet pas d'établir un diagnostic précis sur les gammes de composition du type II. Cependant, il semblerait que la teneur en $\mathrm{MnO}$ soit un des facteurs déterminant. Deux compositions classiques du type III sont données.

La composition chimique de l'analyse $n^{\circ} 1$ correspond à un laitier récent formé de clinopyroxènes alumineux pauvres en $\mathrm{Fe}$ et riches en $\mathrm{Ca}$ et $\mathrm{Mg}$. Les billes de fer métal sont minuscules (Site de Savignac-Lédrier récent), la faible teneur en $\mathrm{Fe}_{2} \mathrm{O}_{3} \mathrm{t}$ traduit leur abondance minime. L'analyse $n^{\circ} 2$ (haut fourneau archaïque de Savignac-Lédrier) correspond à un laitier à clinopyroxènes alumineux riches en $\mathrm{Mg}$ et $\mathrm{Ca}$ et comprenant un peu de $\mathrm{Fe}$. La richesse en $\mathrm{Fe}_{2} \mathrm{O}_{3} \mathrm{t}$ est également due à la concentration du fer dans la matrice vitreuse et à la présence de grosses billes de fer métal. L'analyse $n^{\circ} 3$ (Rogéville) est un laitier archaïque formé de wollastonite n'incorporant pas du tout de fer. Ce dernier se concentre dans la phase vitreuse et dans les billes de métal. L'analyse $n^{\circ} 4$ (Lapphytan) représente un laitier archaïque dont la phase minérale est un feldspath sans fer. Ce dernier élément ce situe encore une fois dans la phase vitreuse et dans les billes de métal. L'analyse $n^{\circ} 5$ (Oulches) correspond à un laitier de bas fourneau à clinopyroxènes riches en $\mathrm{Fe}$ et $\mathrm{Ca}$. Le fer se localise également dans la phase vitreuse et dans les très nombreuses billes de métal qui peuvent être de taille pluri-millimétrique. L'analyse $n^{\circ} 6$ (Frouard) est un quasi-laitier calcique provenant d'un bas fourneau, contenant de la mélilite (incorporant du fer) et de très petites billes de métal. Fe se localise quand même principalement dans la phase vitreuse. 


\begin{tabular}{|c|c|c|c|c|c|c|c|c|c|c|}
\hline & $\begin{array}{c}\mathrm{T} \mathrm{I} \\
-1-\end{array}$ & $\begin{array}{c}\text { T I } \\
-2-\end{array}$ & $\begin{array}{c}\mathrm{T} \text { I } \\
-3-\end{array}$ & $\begin{array}{c}\mathrm{T} \text { I } \\
-4-\end{array}$ & $\begin{array}{c}\text { T I } \\
-5-\end{array}$ & $\begin{array}{c}\mathrm{T} \text { I } \\
-6-\end{array}$ & $\begin{array}{c}\text { T I } \\
-7-\end{array}$ & $\begin{array}{c}\text { T II } \\
-8-\end{array}$ & $\begin{array}{c}\mathrm{T} \text { III } \\
-9-\end{array}$ & $\begin{array}{c}\mathrm{T} \text { III } \\
-10-\end{array}$ \\
\hline $\mathrm{SiO}_{2}$ & 55,32 & 50,93 & 49,68 & 61,25 & 55,41 & 38,28 & 38,30 & 31,93 & 23,61 & 24,35 \\
$\mathrm{Al}_{2} \mathrm{O}_{3}$ & 10,53 & 15,13 & 10,48 & 3,46 & 10,27 & 13,35 & 13,93 & 8,57 & 4,82 & 10,96 \\
$\mathrm{Fe}_{2} \mathrm{O}_{3}$ & 1,04 & 9,91 & 2,16 & & 13,18 & & & 54,06 & 72,02 & 41,90 \\
$\mathrm{FeO}$ & & & & 1,76 & & 7,25 & 39,17 & & & \\
$\mathrm{MnO}$ & 0,86 & 0,88 & 0,26 & 5,63 & 0,50 & 0,90 & 0,72 & 4,82 & 1,12 & 0,44 \\
$\mathrm{MgO}$ & 11,00 & 6,75 & 1,29 & 23,28 & 0,80 & 1,99 & 0,51 & 0,78 & 0,48 & 1,58 \\
$\mathrm{CaO}$ & 18,14 & 11,24 & 29,42 & 3,64 & 17,59 & 32,73 & 4,13 & 2,20 & 2,41 & 17,39 \\
$\mathrm{Na}_{2} \mathrm{O}$ & 0,12 & 0,31 & 0,31 & 0,34 & 0,11 & 0,35 & 0,18 & 0,03 & 0,05 & 0,17 \\
$\mathrm{~K}_{2} \mathrm{O}$ & 1,82 & 1,40 & 2,40 & 0,47 & 1,93 & 1,32 & 0,58 & 1,21 & 0,69 & 0,83 \\
$\mathrm{TiO}_{2}$ & 0,44 & 0,67 & 0,63 & 0,06 & 0,82 & 0,69 & 0,88 & 0,33 & 0,25 & 0,50 \\
$\mathrm{P}_{2} \mathrm{O} 5$ & 0,07 & 0,07 & 0,31 & 0,00 & 0,21 & - & - & 0,45 & 0,40 & 2,72 \\
$\mathrm{PF}^{2}$ & 0,43 & 1,87 & 1,90 & - & $-1,60$ & - & - & $-5,19$ & $-5,87$ & $-1,97$ \\
\hline total & 99,77 & 99,16 & 98,84 & 99,94 & 99,22 & 96,86 & 98,40 & 99,19 & 99,98 & 98,87 \\
\hline
\end{tabular}

Tab. 5 : Exemples d'analyses chimiques des différents types de scories paléosidérurgiques en \% poids oxydes.

-1-laitier de HF récent de Savignac-Lédrier ; -2-3-4- laitier de HF archaĩque de Savignac-Lédrier, de Rogéville et de Lapphyttan ; -5- laitier de BF de Oulches ; -6-quasi-laitier calcique de BF de Frouard ; -7-quasi-laitier siliceux de BF de Bellaires III ; -8- scorie de type II de Oulches ; -9. scorie de type III siliceuse de Oulches ; -10-scorie de type III calcique de Frouard. ((-) non dosé (analyse ponctuelle à la microsonde).

L'analyse $n^{\circ} 7$ (Bellaires III) représente un quasi-laitier siliceux. La richesse en fer est due à la présence de fayalite, spinelle et billes de métal dont la taille peut être assez importante. La teneur élevée en fer de l'analyse $n^{\circ} 8$ (Oulches) est due à la présence des mêmes phases minérales que pour l'échantillon $n^{\circ} 7$. La plus grande quantité de cristaux de fayalite pourrait expliquer la plus faible teneur en fer de la matrice vitreuse. Les scories de type III (analyses $n^{\circ} 9$ (Oulches) et $n^{\circ} 10$ (Frouard)) sont caractérisées par des teneurs en silice plus faibles. Les teneurs en fer peuvent être très importantes s'il n'y a pas de calcium du fait de l'incorporation du fer dans la fayalite. A plus forte teneur en $\mathrm{CaO}$, la mélilite cristallise et une moins grande quantité de fer est incorporée dans les cristaux.

L'attribution typologique des quasi-laitiers pose encore ici un problème. De part leur teneur élevée en fer et la présence de fayalite, ils s'apparentent au type II. Mais par l'abondance des billes de métal et du verre, l'absence de wüstite, la présence de coulure de fonte sur certains sites, ils s'apparentent aux scories de type I. Quasi-laitiers et scories de type II ont souvent été globalement dénommés «scories légères» (i.e. relativement moins denses que les scories de type III). La différence entre les deux types ne peut se faire que grâce à la caractérisation macro et microscopique.

\section{8 - CHIMISME DES SCORIES MARQUÉ PAR L'ÉVOLUTION DES FOURS ET PAR L'UTILISATION D'AJOUTS}

Lorsque des scories présentent de fortes teneurs en $\mathrm{CaO}$, il est souvent difficile de savoir si cette richesse est due à un apport volontaire de castine, ou à l'utilisation d'un minerai calcique. Le but de ce travail est de concevoir un diagramme permettant de distinguer l'apport calcique d'un ajout de castine de celui d'un minerai, par l'utilisation des analyses chimiques globales. Le diagramme $\mathrm{Fe}-(\mathrm{Ca}+\mathrm{Mg}) /$ $\mathrm{Si}+\mathrm{Al}$ convient parfaitement pour cela. Il met également en évidence l'évolution chimique des scories avec celle des fours lorsqu'il n'y a pas eu d'ajouts de castine.

La teneur en fer dans les scories étudiées dans ce travail, varie de manière très importante de $85 \%$ poids d'oxydes $\mathrm{Fe}_{2} \mathrm{O}_{3} \mathrm{t}$ dans les scories denses de Villejuif à $0 \%$ poids dans des laitiers de Savignac-Lédrier récent et dans les laitiers modernes, de type Moulaine. Nous introduisons la notion de taux de récupération du fer afin de marquer le «rendement» du four. Un taux de récupération faible (donc une forte teneur en fer dans la scorie) caractérise un four d'évolution technique peu avancé. Attention, ceci peut marquer également une mauvaise conduite d'un four ayant normalement un bon taux de récupération. L'évolution de la teneur en fer se fait selon trois chemins schématisés sur la figure 5 .

Le trajet A montre l'évolution de la teneur en fer dans un système très peu riche en $\mathrm{CaO}+\mathrm{MgO}$, sans ajout de castine. Il y a augmentation de la teneur en $\mathrm{SiO}_{2}+\mathrm{Al}_{2} \mathrm{O}_{3}$ alors que la teneur en $\mathrm{Fe}_{2} \mathrm{O}_{3} \mathrm{t}$ baisse. Toutes ces scories proviennent de bas fourneaux. L'évolution se fait depuis les scories denses fayalitiques (qui peuvent être considérées comme les matériaux résultant du taux de récupération le plus faible) jusqu'aux quasi-laitiers, les scories de type II se localisant entre ces deux pôles. La quantité de fer restant dans la scorie est diminuée par un meilleur contrôle dans la conduite du four : plus forte température, meilleure ventilation etc... Elle atteint un minima de l'ordre de $14 \%$ poids de $\mathrm{Fe}_{2} \mathrm{O}_{3}$, qu'il ne semble pas possible de dépasser.

Le trajet B montre l'évolution de la teneur en fer dans un système bas fourneau sans ajout de castine, mais où la richesse en $\mathrm{Ca}$ est due au minerai lui-même (exemple de la Minette de Lorraine). L'augmentation conjointe de $\mathrm{SiO}_{2}+\mathrm{Al}_{2} \mathrm{O}_{3}$ et de $\mathrm{CaO}+\mathrm{MgO}$ est correlée à la baisse de la teneur en FeO. La charge est alors principalement composé de $\mathrm{SiO}_{2}, \mathrm{CaO}$ et $\mathrm{Fe}_{2} \mathrm{O}_{3}$ t, et en plus faible quantité, $\mathrm{Al}_{2} \mathrm{O}_{3}$. De part la composition chimique du minerai, il est inutile de faire un ajout de castine.

Le trajet C1 montre l'évolution de la teneur en fer dans des fours (bas et hauts fourneaux) lorsqu'il y a eu un ajout de castine à la charge. L'augmentation de la teneur en $\mathrm{CaO}+\mathrm{MgO}$ est alors correlée à la baisse de la teneur en $\mathrm{Fe}_{2} \mathrm{O}_{3} \mathrm{t}$. La teneur en $\mathrm{SiO}_{2}+\mathrm{Al}_{2} \mathrm{O}_{3}$ baisse très faiblement. Les laitiers les plus riches en fer proviennent de bas fourneaux. Les laitiers pauvres en fer, proviennent de hauts fourneaux récents. Les laitiers de hauts fourneaux archaïques et les quasi-laitiers calciques de bas fourneaux (Frouard) se situent en position intermédiaire sur cet alignement. La quantité de chaux est augmentée régulière- 

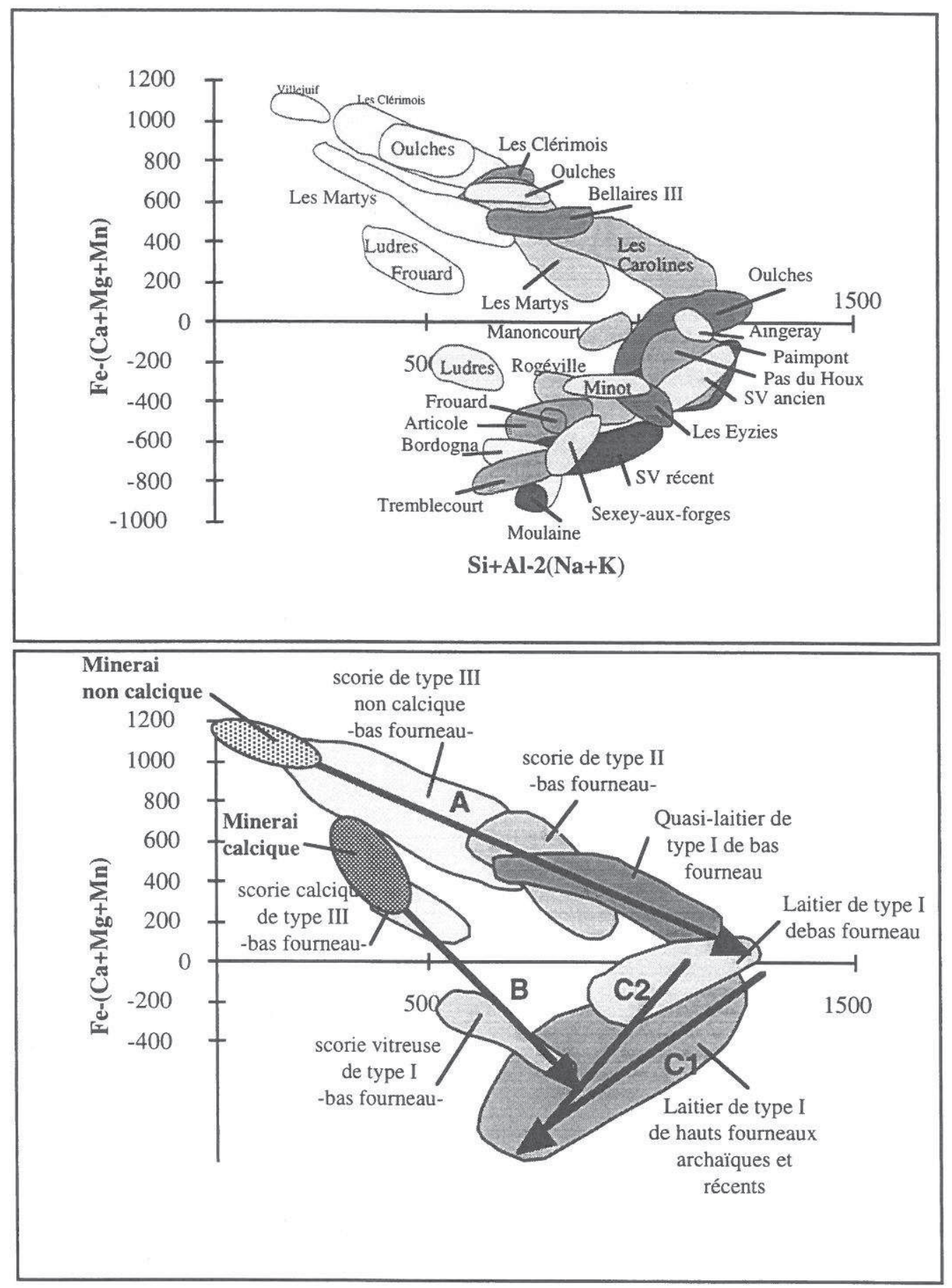

Fig. 5 : Localisation des scories de la sidérurgie dans le diagramme $\mathrm{Fe}-(\mathrm{Ca}+\mathrm{Mg}+\mathrm{Mn})$ en fonction de $\mathrm{Si}+\mathrm{Al}$ et évolution de la teneur en fer de celles-ci. Trajet A : évolution dans un système bas fourneau, avec utilisation d'un minerai pauvre en $\mathrm{Ca}$ et sans ajout de castine. Trajet B : évolution dans un système bas fourneau, avec utilisation d'un minerai calcique et sans ajout de castine. Trajet $\mathrm{Cl}$ : évolution dans un système haut fourneau, avec utilisation de mélanges minerais non calciques/castine. Trajet $\mathrm{C} 2$ : évolution dans un système haut fourneau, avec utilisation de minerais calciques.

ment dans la charge. On passe d'une réduction en bas fourneau (teneurs en $\mathrm{Fe}_{2} \mathrm{O}_{3} \mathrm{t}$ les plus fortes) à une réduction en haut fourneau pour des teneurs en $\mathrm{Fe}_{2} \mathrm{O}_{3}$ t proches de zéro. L'utilisation d'un minerai calcique en haut fourneau semblerait conduire à une évolution légèrement décalée de la teneur en fer (scories de Manoncourt, Sexeyaux-Forges, Rogéville et Tremblecourt) selon le trajet C2. Des analyses sur des sites complémentaires seraient nécessaires pour confirmer cette observation.

Ce diagramme chimico-minéralogique (fig. 5) met en évidence la différence de composition de la charge de départ : soit minerai calcique, soit minerai «siliceux», soit minerai «siliceux» avec ajout de castine. L'évolution chimique des scories dans chaque lignée est essentiellement due à l'amélioration technique des fours et à une meilleure conduite de ceux-ci lors des opérations de réduction. Il est possible d'observer les limites de chaque système, ainsi dans des fours chargés de minerais "siliceux» sans ajouts de castine, il reste une teneur résiduelle en fer de $14 \%$. Pour descendre en dessous de cette valeur, il faut ajouter de la castine. De même, l'évolution des techniques des bas fourneaux chargés avec des minerais calciques uniquement ne 
permet pas d'obtenir des scories avec des teneurs en fer en dessous de $7 \%$ environ. Là encore, un ajout de castine est indispensable, et pour pouvoir fondre l'ensemble de la charge (qui a une température de fusion plus importante), il devient nécessaire d'utiliser un haut fourneau.

Les contaminations par les parois des fours ou par les cendres ne sont pas visibles sur ce diagramme (fig. 5). En effet, la variation de teneur en $\mathrm{Ca}$ est mise en opposition de celle de $\mathrm{Fe}$. La variation de teneur en Fe est généralement trop importante et «écrase» celle de $\mathrm{Ca}$. Pour estimer la quantité de $\mathrm{Ca}$ apporter dans le système, il faut utiliser d'autres diagrammes, comme cela a été le cas pour les scories de Oulches (Mahé-Le Carlier et al., 1998). Dans un diagramme triangulaire $\mathrm{Si}-\mathrm{Al}-\mathrm{Ca}$, ont été placé les points représentatifs des minerais et des scories. Il a été établi qu'il y avait pu avoir jusqu'à $10 \%$ en poids d'ajouts de castine. La mise en évidence d'une contamination peut se faire par l'utilisation du même diagramme. En plaçant les points représentatifs des minerais et des scories du site des Martys, on peut voir qu'il y a un enrichissement des scories par rapport au minerai de 2 à $3 \%$ poids de $\mathrm{CaO}$. Ceci peut être dû aux cendres. Il reste à déterminer quel est l'enrichissement maximum possible avec les œendres et les parois, afin de pouvoir connaître le seuil à partir duquel on pourra réellement affirmer qu'il y a eu ajout de castine ; pour ce faire les analyses de cendres seraient à multiplier.

\section{CONCLUSION}

L'étude macroscopique, microscopique et chimique des scories de la sidérurgie ancienne permet une caractérisation typologique de celles-ci et une classification en fonction des processus physico-chimiques conduisant à l'apparition du fer métal. Cette typologie est néanmoins liée au type de four utilisé : les scories de type II et III proviennent de bas fourneaux. Les scories de type I peuvent provenir de bas fourneaux (quasi laitiers, parfois laitiers) mais surtout de hauts fourneaux. La caractérisation effectuée permet de proposer une origine pour ces scories si aucun lien n'a pu être fait avec un type de four.

La connaissance des compositions chimiques permet en outre de pouvoir déterminer dans de nombreux cas, et en particulier dans les cas de bas fourneaux, s'il y a eu utilisation de minerais calciques ou s'il y a eu ajouts de castine dans le four.

La forte orientation de l'étude vers les scories vitreuses (Mahé-Le Carlier, 1997) a permis de s'intéresser à un problème non encore totalement résolu : la transition bas fourneau - haut fourneau. Ce travail a montré, par le biais de la connaissance des déchets, qu'il était possible de faire fonctionner certains bas fourneaux en mode indirect, c'est-àdire comme les hauts fourneaux, et ceci dès le troisième siècle après J. -C.

\section{BIBLIOGRAPHIE}

BELHOSTE, J.F., 1986 - The diffusion of the Blast Furnace Process across Western France in the 15 th and 16th Centuries, Jernkontorets Forskning, 39, 5-19.

BENOIT, P., GUILLOT, I., PLOQUIN, A. et FLUZIN, P., 1991 Archéologie et paléométallurgie des sites de Minot et Fontenay en Bourgogne. in : Archaometallurgie Von Kupfer Und Eisen in Westeuropa, September 1986, Mainz, 12-15.

DIEUDONNE-GLAD, N., 1991 - Le Latté à Oulches (Indre). Rapport de fouille programmée, $24 \mathrm{p}$.
DIEUDONNE-GLAD, N., 1993 - Le Latté à Oulches (Indre). Rapport de fouille programmée, $19 \mathrm{p}$.

DUBOIS, C. et PIZANO, L., 1992 - Site de "La Forge» de Savignac-Lédrier. Rapport de fouille de sauvetage de juillet 1992 , Service Régional d'Aquitaine, 80 p.

DUNIKOWSKY, C. et CABBOI, S., 1995 - La sidérurgie chez les Sénons : les ateliers celtiques et gallo-romains des Clérimois (Yonne), $D A F, \mathbf{5 1}, 186$ p.

GSAF (Groupe de Travail Suisse d'Archéologie du Fer) 1991 Technique des fouilles.

JARRIER, C., 1994 - Minéralogie et géochimie de résidus sidérurgiques romains des Martys (Montagne Noire, Aude) : Approche des conditions de fonctionnement de la réduction directe. In : Mangin dir. La sidérurgie ancienne de l'Est de la France dans son contexte européen, Besançon, 10.13 Novembre 1993, 57 64.

JARRIER, C., ANDRIEUX, P., DOMERGUE, C., PIERAGGI, B., PLOQUIN, A., TOLLON, F., 1997 - Elaboration du fer par réduction directe : essais de reproduction des procédés antiques. La revue de métallurgie-CIT/Science et Génie des Matériaux, 691-704.

KNAU, H.L., 1994 - Renn- und Roheisenverhattung, Osemundfrischen Zur Geschichte der Eisen- und Stahlerzeugnung im märkischen Sauerland, 165-171. In : Mangin dir. La sidérurgie ancienne de l'Est de la France dans son contexte européen, Besançon, 10-13 Novembre 1993.

LEROY, M., 1993 - La sidérurgie ancienne en Lorraine avant le haut fourneau : Etude du développement historique et des conditions techniques de l'utilisation du minerai oolithique lorrain (la Minette) en métallurgie de réduction directe. Thèse Besançon, 434 p. Publication CNRS 1997, après légères adaptations.

LEROY, M. et PLOQUIN, A., 1994 - Approche archéométrique de la proto-industrie du fer : Les conditions de réduction des minerais de fer de Lorraine et la chaîne opératoire dans la sidérurgie antérieure à l'apparition du haut fourneau. Revue d'Archéométrie, 18, 53-64.

MAGNUSSON, G., 1996 - Ironmaking in a long time perspective some important questions, 29-40, in Magnusson ed., The importance of Ironmaking. Technical Innovation and Social Change. Norberg Conference, may 1995. Jernkontorets Bergshistoriska Utskott, H58, H62.

MAHÉ-LE CARLIER, C., 1997 - Caractérisation pétrographique et chimique d'analogues de déchets vitrifiés actuels: les scories de la métallurgie ancienne. Etude de l'altération naturelle et expérimentale. Thèse INPL Nancy, 337 p.

MAHÉ-LE CARLIER, C., DIEUdONNE-GLAD, N., PLOQUIN, A., 1998 - Des laitiers obtenus dans un bas fourneau ? Etude chimique et minéralogique des scories du site de Oulches (Indre), Revue d'Archéométrie, 22, 91-101.

MEYER-ROUDET, H., 1999 - A la recherche du métal perdu, Errance, $192 \mathrm{p}$

PLOQUIN, A., 1993 - A propos des scories légères et laitiers associés aux déchets paléosidérurgiques en France, quelques apports de la base de données Artémise-scories. in Bloomery IronMaking during 2000 years, Budalen 1991, 91-103.

PLOQUIN, A., 1994 - L'analyse chimique en paléosidérurgie. In : Mangin dir. La sidérurgie ancienne de l'Est de la France dans son contexte européen, Besançon, 16-23 Novembre 1993.

PLOQUIN, A., MAHE, C., LEROY, M., DIEUDONNÉ-GLAD, N. and JARRIER, C., 1996 - Reconstruction of Ironmaking Procedure : A Progress Report about Petrographical Studies of Slaggy Wastes from Archaeo-Ironmaking Sites. In : The Importance of Ironmaking. Technical Innovation and Social Change, Norberg (Suède) 1995, 2, ed. Gert Magnusson, 2, $105-119$.

SERNEELS, V., 1993 - Archéométrie des scories de fer : Recherches sur la sidérurgie ancienne en Suisse Occidentale. Thèse Lausanne, Suisse, 327 p.

TYLECOTE, R.F., 1987 - The Early History of Metallurgy in Europe. Longman Archaeology Series, London, 391 p.

WALDBAUM, J.C., 1980 - The first Archaeological Appearance of Iron and the Transition to the Iron Age, in : Wertime T.A. and Muhly J.D. eds, The Coming of the Age of Iron, Londres, 555 p. 